\title{
Hairy black hole stability in AdS, quantum mechanics on the half-line and holography
}

\author{
Andrés Anabalón, ${ }^{a}$ Dumitru Astefanesei ${ }^{b}$ and Julio Oliva ${ }^{c}$ \\ ${ }^{a}$ Departamento de Ciencias, Facultad de Artes Liberales y \\ Facultad de Ingeniería y Ciencias, Universidad Adolfo Ibáñez, \\ Av. Padre Hurtado 750, Viña del Mar, Chile \\ ${ }^{b}$ Instituto de Física, Pontificia Universidad Católica de Valparaíso, \\ Casilla 4059, Valparaiso, Chile \\ ${ }^{c}$ Departamento de Física, Universidad de Concepción, \\ Casilla 160-C, Concepción, Chile \\ E-mail: andres.anabalon@uai.cl, dumitru.astefanesei@ucv.cl, \\ julio.oliva@uach.cl
}

ABSTRACT: We consider the linear stability of 4-dimensional hairy black holes with mixed boundary conditions in Anti-de Sitter spacetime. We focus on the mass of scalar fields around the maximally supersymmetric vacuum of the gauged $\mathcal{N}=8$ supergravity in four dimensions, $m^{2}=-2 l^{-2}$. It is shown that the Schrödinger operator on the half-line, governing the $S^{2}, H^{2}$ or $\mathbb{R}^{2}$ invariant mode around the hairy black hole, allows for nontrivial self-adjoint extensions and each of them corresponds to a class of mixed boundary conditions in the gravitational theory. Discarding the self-adjoint extensions with a negative mode impose a restriction on these boundary conditions. The restriction is given in terms of an integral of the potential in the Schrödinger operator resembling the estimate of Simon for Schrödinger operators on the real line. In the context of AdS/CFT duality, our result has a natural interpretation in terms of the field theory dual effective potential.

KEYwords: Black Holes, AdS-CFT Correspondence

ARXiv EPrint: 1507.05520 


\section{Contents}

1 Introduction 1

$\begin{array}{lll}2 & \text { Linear stability } & 3\end{array}$

3 Asymptotic effective potential $\quad 5$

$\begin{array}{lll}4 & \text { A necessary condition for stability } & 6\end{array}$

5 Holography and effective potentials $\quad 8$

6 Conclusions $\quad 9$

\section{Introduction}

A form of understanding the asymptotically flat no-hair theorems is that they connect the stability of the ground state and the existence of hairy black holes. Namely, if the relevant scalar-tensor theory has a stable Minkowski vacuum then it does not admit hairy black hole configurations. Indeed, the Bekenstein no-hair theorem shows that a convex scalar field potential implies the non-existence of hairy black hole solutions [1]. The same has been shown to be true for non-negative potentials [2, 3]. Moreover, it is now clear that a necessary condition for the existence of asymptotically flat hairy black hole solutions is a scalar field potential with a negative region [4-7]. Therefore, if the scalar field potential has a extremum allowing for a Minkowski vacuum, where the scalar field potential necessarily vanishes, a sufficiently large perturbation will naturally explore the sector where the potential is negative, destabilizing the Minkowski ground state. Hence, one may wonder whether the hairy black hole itself is stable. However, it happens that all the asymptotically flat hairy black holes described in the literature are unstable under spherically symmetric perturbations [8-12]. On the contrary, odd-parity perturbations are generically well behaved around hairy black holes [13]. For a recent review of the asymptotically flat case see [14]. Indeed, one is having in mind here a single, real scalar field. Already the case of a complex scalar field is different. The black holes no-hair theorems can be skipped in this case because the scalar field and the metric do not share the same symmetries (but the energy momentum tensor and the metric do) $[15,16]$.

However, for a single and real scalar field, the picture is completely different in asymptotically AdS spacetimes in the sense that it is not necessary to have a positive scalar field potential to have a stable AdS vacuum. As is well-known, scalar fields with Dirichlet boundary conditions and masses above the Breitenlohner-Freedman (BF) bound,

$$
m_{B F}^{2}=-\frac{(D-1)^{2}}{4 l^{2}}
$$


where $D$ is the spacetime dimension and $l$ is the AdS radius, do not generate linear instabilities in global AdS spacetimes [17, 18].

When the squared scalar field mass, $\mathrm{m}^{2}$, is above (or saturates) the BF bound and strictly less than the unitarity bound,

$$
m_{B F}^{2} \leq m^{2}<m_{B F}^{2}+l^{-2},
$$

the Klein-Gordon operator has non-trivial self-adjoint extensions in global AdS spacetime [19] (for a pedagogical discussion of self-adjoint extensions see [20]). Each of these self-adjoint extensions defines a class of theories and some of these theories are equivalently defined by the requirement of the existence of a soliton with a given value of the scalar field at the origin. Such constructions go by the name of designer gravity [21]. Furthermore, these generalized boundary conditions are present for all examples of hairy black holes known so far [22-46].

There is a no-hair conjecture proposed by Hertog [47] that, modulo certain technical requirements, captures the spirit of the asymptotically flat case. It states that, if there exists a suitable superpotential that is globally defined, the boundary conditions are AdS invariant, and there is a bound of the energy, ${ }^{1}$ then the theory does not allows the existence of hairy black holes. Global AdS has vanishing mass for all the boundary conditions, hence it should saturate the bound and, if there is no hairy soliton, its non-perturbative stability would be ensured. Within the conditions of the conjecture, non-perturbative stability of the ground state imply the no-hair condition.

In this paper we shall consider the less explored subject of hairy black hole stability in asymptotically AdS spacetimes. The theory of black hole stability was developed by Regge-Wheeler and Zerilli $[51,52]$. The higher dimensional generalization was done by Ishibashi-Kodama [53]. The approach to the problem is to linearize the system around a background and to classify the perturbations as representations of the background isometries. The dynamics for each type of perturbation decouples satisfying a wave equation with an effective potential. It is straightforward to show that, in the frequency domain, the modes satisfy a Schrödinger equation. When the spectrum is positive and the relevant domain of the operator is $L^{2}(\mathbb{R})$, it has a unique self-adjoint extension, namely the Friedrichs extension, which implies that the linearized evolution is well defined and the background is stable, at least in the static case [54].

AdS is not globally hyperbolic, which means that to make sense of the evolution it is necessary to provide the initial data and impose certain boundary conditions. Namely, around a black hole background, the Schrödinger operator governing the dynamics acts on $\left.\left.L^{2}(]-\infty, 0\right]\right)$. Hence, it has an infinite number of self-adjoint extensions parameterized by a real number. For the case at hand, and even if the effective potential is everywhere positive, the self-adjoint extensions modify the spectrum of the Schrödinger operator introducing exactly one negative eigenvalue. As we shall see below, these non-trivial self-adjoint extensions are associated with the existence of the two normalizable modes in the window (1.2). The main objective of this paper is to establish which boundary conditions implies the

\footnotetext{
${ }^{1}$ Mixed boundary conditions introduce subtleties to the existence of a bound [48], see [49, 50].
} 
existence of this instability, thus providing a simple tool to analyze a complex problem. The condition is sufficient and, if one would be interested in finding a stable hairy black hole, it allows to reduce the number of cases relevant to study in the first place.

Whenever the gravitational theory has a CFT dual and the boundary conditions are AdS invariant, they correspond to multi-trace deformations of the boundary CFT [55] — for a nice discussion with the detailed holographic dictionary, see [56]. As formulated in [21], the non-perturbative stability of AdS corresponds to a convex potential in the field theory with only one global minimum. If the effective potential has several global minima, with zero energy, they are identified with the existence of hairy solitons. A clear holographic picture thus arises connecting stability of the gravitational theory and of the field theory. Furthermore, the dual interpretation of hairy black holes (and their stability) in terms of a finite temperature version of the field theory effective potential was first put forward and analyzed in [57]. Indeed, our condition for hairy black hole instability seems to be suited for an interpretation along the lines of $[21,57]$ and we comment on it. This shows a clear connection between the mechanical stability of hairy black holes and the study of the positive energy theorems. There has been a lot a of work on proving positive energy theorems for certain classes of designer gravity theories. The first theorem for bounded effective potentials were proven in [58] and this was generalized to certain classes of effective potential that are even unbounded below in [48].

This kind of instability at the conformal mass has been already observed by Martinez [59] in the particular case of the Martinez-Zanelli hairy black hole [60], whose source is a conformally coupled scalar field in three dimensions. The Schrödinger operator governing the evolution of the linearized dynamics has an everywhere non-negative effective potential around the Martinez-Zanelli hairy black hole, however a normalizable mode that is exponentially growing in time is shown to exist. Our analysis sheds light on this counterintuitive result.

\section{Linear stability}

The theory of the linear spherically symmetric perturbations in the case of gravity coupled to scalar fields dates back to [8], more recently it has been discussed in [9-12]. Here, we provide a straightforward generalization to the hyperbolic and planar modes. The action principle is given by

$$
S\left[g_{\mu \nu}, \phi\right]=\int d^{4} x \sqrt{-g}\left[\frac{R}{2 \kappa}-\frac{1}{2}(\partial \phi)^{2}-V(\phi)\right] .
$$

In this section $V(\phi)$ is an arbitrary function of $\phi$ with at least one AdS vacuum. The field equations are

$$
E_{\mu \nu}=R_{\mu \nu}-\frac{1}{2} g_{\mu \nu} R-\kappa T_{\mu \nu},
$$

When the Einstein equations are satisfied, the scalar field equation holds as a consequence of the conservation of the energy momentum tensor

$$
T_{\mu \nu}=\partial_{\mu} \phi \partial_{\nu} \phi-g_{\mu \nu}\left[\frac{1}{2}(\partial \phi)^{2}+V(\phi)\right] .
$$


Let us consider the four dimensional time dependent metric

$$
d s^{2}=-\Delta_{1}(x, t) d t^{2}+\Delta_{2}(x, t) d x^{2}+C(x, t) d \Sigma_{k},
$$

where $d \Sigma_{k}$ is the metric of a two dimensional space of constant curvature, with Ricci scalar normalized to $2 k$, and the scalar field

$$
\phi=\phi(x, t) .
$$

It is always possible to use the differmorphism invariance to set $C(x, t)=C(x)$, where, for the sake of generality, we choose to let $C(x)$ arbitrary. We are interested in studying the linearized dynamics around a background solution. Hence, we introduce the expansion

$$
\begin{aligned}
\Delta_{1}(x, t) & =A(x)+\epsilon A_{1}(x, t), \\
\Delta_{2}(x, t) & =B(x)+\epsilon B_{1}(x, t), \\
\phi(x, t) & =\phi_{0}(x)+\epsilon \phi_{1}(x, t), \\
V(\phi) & =V_{0}+\epsilon V_{1} \phi_{1}(x, t),
\end{aligned}
$$

where

$$
V_{0}=V\left(\phi_{0}\right), \quad V_{n}=\left.\frac{d^{n} V}{d \phi^{n}}\right|_{\phi=\phi_{0}} .
$$

From now on, we shall assume that the Einstein field equations are solved by the $\epsilon=0$ set of functions. Birkhoff's theorem ensures that all the dynamics is driven by the scalar field. Indeed, it is possible to write the metric perturbations in terms of the $\phi_{1}(x, t)$ by using the Einstein field equations. Let us consider first:

$$
E_{t x}=\left(\frac{C^{\prime}}{2 B C} \dot{B}_{1}-\kappa \dot{\phi}_{1} \phi_{0}^{\prime}\right) \epsilon+O\left(\epsilon^{2}\right)
$$

where $\dot{\phi}_{1}=\partial_{t} \phi_{1}$ and $\phi_{1}^{\prime}=\partial_{x} \phi_{1}$. Thus, modulo a trivial redefinition of the perturbations it follows from (2.11) that

$$
B_{1}(x, t)=2 \kappa \frac{C B}{C^{\prime}} \phi_{1} \phi_{0}^{\prime},
$$

We replace (2.12) in the remaining Einstein equations, then automatically follows that $E_{t t}=O\left(\epsilon^{2}\right)$. The radial equation is also a simple constraint

$E_{x x}=\left(-\frac{1}{2} \frac{C^{\prime} A^{\prime}}{C A^{2}} A_{1}+\frac{1}{2} \frac{C^{\prime}}{C A} A_{1}^{\prime}+\kappa \frac{B}{C^{\prime}}\left[-2 k \phi_{0}^{\prime}+V_{1} C^{\prime}+2 \kappa C V_{0} \phi_{0}^{\prime}\right] \phi_{1}-\kappa \phi_{0}^{\prime} \phi_{1}^{\prime}\right) \epsilon+O\left(\epsilon^{2}\right)$.

Hence, it is possible to obtain $A_{1}^{\prime}$ from (2.13). When $A_{1}^{\prime}$ and $B_{1}$ are replaced in the equation along the coordinates of the sphere a messy expression is obtained. However, this equation can be simplified by introducing a master variable

$$
\psi(z, t)=\phi_{1}(x, t) C(x)^{1 / 2},
$$

where $z$ is the tortoise coordinate

$$
d z=\left(\frac{B}{A}\right)^{1 / 2} d x
$$


The master equation is

$$
-\partial_{z}^{2} \psi+U \psi=-\partial_{t}^{2} \psi
$$

with the effective potential

$$
\frac{U}{A}=4 \kappa C\left[\left(\kappa V_{0} C-k\right)\left(\frac{d \phi_{0}}{d C}\right)^{2}+V_{1}\left(\frac{d \phi_{0}}{d C}\right)\right]-\kappa V_{0}+V_{2}+\frac{k}{C}-\frac{1}{4 B}\left(\frac{C^{\prime}}{C}\right)^{2} .
$$

If $z$ takes its values in the whole real line and $U$ is non-negative, the operator (2.16) is essentially self-adjoint and its spectrum is positive which implies that the background is mode stable under spherically symmetric perturbations. ${ }^{2}$ It is possible to extract some generic behaviour of $U$ if the asymptotic form of the theory is specified.

\section{$3 \quad$ Asymptotic effective potential}

We shall consider a potential that yields the following expansion around the AdS vacuum

$$
V=-\frac{3}{\kappa l^{2}}+\frac{1}{2} m^{2} \phi^{2}+\xi \phi^{4}+O\left(\phi^{5}\right) .
$$

We have intentionally omitted a cubic term in the self-interaction, as its inclusion makes the analysis more complicated due to the existence of subleading logarithmic branches [62]. Evaluating (2.17) in the AdS background with coordinates $C=r^{2}, A=\frac{r^{2}}{l^{2}}+k=B^{-1}$ we get $^{3}$

$$
U=\left(m^{2}+\frac{2}{l^{2}}\right)\left(\frac{r^{2}}{l^{2}}+k\right)
$$

For masses above $m^{2}=-2 l^{-2}$ the potential $U(2.17)$ is asymptotically positive and divergent. For masses below this one, the potential $U$ is asymptotically unbounded from below [61]. However, when $m^{2} \geq m_{B F}^{2}$ and for suitable boundary conditions the spectrum can be positive. The case $m^{2}=-2 l^{-2}$ is the limiting case and we shall focus on it from now on. As it has been worked out in detail in [25, 62], in this case the scalar field fall-off is

$$
\phi=\frac{\alpha}{r}+\frac{\beta}{r^{2}}+O\left(r^{-3}\right)
$$

where $\alpha$ and $\beta$ denote two functions of the other coordinates. The relevant fall-off of the metric is

$$
\begin{aligned}
-g_{t t} & =\frac{r^{2}}{l^{2}}+k+O\left(r^{-1}\right), \\
g_{m n} & =r^{2} h_{m n}+O\left(r^{-1}\right), \\
g_{r r} & =\frac{l^{2}}{r^{2}}-\frac{\left(l^{4} k+\kappa \alpha^{2} l^{2} / 2\right)}{r^{4}}+O\left(r^{-5}\right),
\end{aligned}
$$

\footnotetext{
${ }^{2}$ Non-modal stability is harder to prove and just recently has been confirmed for the Schwarzschild black hole [63].

${ }^{3}$ For obvious reasons we change the notation from $x$ to $r$ in this section.
} 
Here, $h_{m n}\left(x^{m}\right)$ is the two-dimensional metric associated to the sphere, plane or locally hyperbolic space of constant Ricci scalar $2 k, d \Sigma_{k}$. The asymptotic value of the potential is

$$
U_{0}:=\lim _{r \rightarrow \infty} U=\frac{3}{2} \frac{\alpha^{2}\left(8 \xi l^{2}+\kappa\right)}{l^{4}} .
$$

Hence, we see that the quartic term governs the asymptotic value of $U$ when the slow branch is on. As we have just discussed the effective potential for massless perturbations, $m^{2}=-2 l^{-2}$, vanishes in local AdS spacetime (3.2). However, the same effective potential evaluated in asymptotically local AdS spacetime is not asymptotically zero.

As is standard, the tortoise coordinate $(2.15)$ goes to $-\infty$ at the black hole horizon and can be set to be zero at the AdS boundary. Hence, the relevant Schrödinger operator has non-trivial self-adjoint extensions and stability becomes subtler.

\section{A necessary condition for stability}

As the subject of self-adjoint extensions is not so well-known we would like to start this section with a short heuristic discussion. Let us consider the operator $H=-\partial_{z}^{2}$. If $z$ belongs to the half real line, $z \in]-\infty, 0]$, then $H$ has a square integrable eigenfunction with a negative eigenvalue,

$$
\begin{aligned}
& \psi=\exp (\lambda z), \\
& \psi=\exp (\lambda z) \Longrightarrow H \psi=E \psi=-\lambda^{2} \psi .
\end{aligned}
$$

with $\lambda>0$. The existence of this eigenvalue is related to the boundary conditions that the eigenfunction has. It satisfies mixed boundary conditions

$$
b \psi(0)=a \psi^{\prime}(0)
$$

with

$$
\lambda=\frac{b}{a} .
$$

$a=0$ is the Dirichlet boundary condition, $b=0$ is the Neumann boundary condition and any other combination are Robin boundary conditions. In this case, $H$ admits a oneparameter family of self adjoint extensions, parameterized by $\lambda$. It is easy to see that the negative eigenvalue only exists for Robin boundary conditions. Furthermore, $\psi$ is square integrable only when the ratio of $a$ and $b$ is positive. Still, when $\lambda<0$ the self-adjoint extensions exists but the negative energy state is excluded from the spectrum.

Let us consider now the operator $\mathcal{H}=-\partial_{z}^{2}+U$, defined on the half real line as before and let us consider a, square integrable, energy eigenstate $\psi$. It satisfies

$$
\mathcal{H} \psi=E \psi \Longrightarrow \psi \mathcal{H} \psi=E \psi^{2},
$$

Integrating by parts (4.5) yields

$$
-\left.\psi \partial_{z} \psi\right|_{-\infty} ^{0}+\int_{-\infty}^{0}\left[\left(\partial_{z} \psi\right)^{2}+U \psi^{2}\right] d z=E \int_{-\infty}^{0} \psi^{2} d z
$$


Using the boundary conditions and the fact that $\psi$ is a bound state, $\psi(-\infty)=0,(4.6)$ yields

$$
-\lambda \psi(0)^{2}+\int_{-\infty}^{0}\left[\left(\partial_{z} \psi\right)^{2}+U \psi^{2}\right] d z=E \int_{-\infty}^{0} \psi^{2} d z
$$

As in the case where the potential vanishes we see that $U \geq 0$ and $\lambda \leq 0$ automatically excludes negative energies. Moreover, whenever $U \geq 0$, the l.h.s. integral in (4.7) seems to suggest that it is still possible to have a positive spectrum for $\lambda>0$; introducing a competition between the potential, $U$, and the self-adjoint extension parameter $\lambda$.

Indeed, let us suppose that the spectrum of $\mathcal{H}$ is positive with $U \geq 0$ and $\lambda>0$. As is well-known, given the zero-energy Schrödinger operator

$$
\mathcal{H} \chi=0
$$

the number of nodes of $\chi$, count the number of bound states of $\mathcal{H}$. The linearity of the problem allows to set the boundary conditions in the simplified form

$$
\chi(0)=1, \quad \chi^{\prime}(0)=\lambda .
$$

We shall assume that $\chi$ has no node. Hence, there must be a $z_{0}$, where $\chi^{\prime}\left(z_{0}\right)=0$. Notice that $U>0 \Longleftrightarrow \chi^{\prime \prime}>0 \Longrightarrow z_{0}$ is a local minimum of $\chi$. The integral of (4.8) between $z_{0}$ and 0 yields

$$
\lambda=\int_{z_{0}}^{0} U \chi d z
$$

A bound on $\lambda$ then follows from

$$
\int_{z_{0}}^{0} U \chi d z<\chi(0) \int_{z_{0}}^{0} U d z=\int_{z_{0}}^{0} U d z<\int_{-\infty}^{0} U d z .
$$

A necessary condition for stability is then

$$
\lambda<\int_{-\infty}^{0} U d z
$$

In terms of the original perturbation

$$
\phi_{1}=\frac{\alpha_{1}}{r}+\frac{\beta_{1}}{r^{2}}+O\left(r^{-3}\right)
$$

$\psi(0)=\alpha_{1}, \psi^{\prime}(0)=-\beta_{1} l^{-2}$ we get

$$
\lambda=-\frac{\beta_{1}}{\alpha_{1} l^{2}} .
$$

It is now clear that not all the boundary conditions allow for linear stability due to the existence of non-trivial self-adjoint extensions. Indeed, if the original scalar-tensor theory has either Dirichlet or Neumann boundary conditions the equation for the perturbation does not admit self-adjoint extensions. When the prescribed dynamics is in terms of Robin boundary conditions, the boundary condition of the perturbation can be read-off from the following equality

$$
\phi=\frac{\alpha}{r}+\frac{\beta(\alpha)}{r^{2}}+O\left(r^{-3}\right)=\phi_{0}(r)+\epsilon \phi_{1}(r, t)
$$


Indeed, using that the background configuration is such that

$$
\phi_{0}=\frac{\alpha_{0}}{r}+\frac{\beta\left(\alpha_{0}\right)}{r^{2}}+O\left(r^{-3}\right)
$$

it follows that the perturbation satisfy

$$
\phi_{1}=\frac{\alpha_{1}}{r}+\frac{\alpha_{1} \beta^{\prime}\left(\alpha_{0}\right)}{r^{2}}+O\left(r^{-3}\right) .
$$

Thus, comparing (4.12), (4.13), (4.14) and (4.17) we find that our necessary condition for stability, in terms of the boundary condition of the scalar-tensor theory is

$$
\beta^{\prime}\left(\alpha_{0}\right)+l^{2} \int_{-\infty}^{0} U d z>0
$$

We could have written the bound in terms of $\int_{z_{0}}^{0} U d z$, however in practice is much more useful the bound in terms of the full integral.

When the Schrödinger operator is defined in the whole real line there is an estimate of the value of the lowest eigenvalue due to Simon [65]. It can also be seen as a necessary condition, for the positivity of the spectrum:

$$
\int_{-\infty}^{\infty} U d z>0
$$

Our result may be seen as a generalization of it.

\section{$5 \quad$ Holography and effective potentials}

In the dual field theory, $\alpha$ represents the expectation value of the operator that condensates in the hairy black hole states. Effective potentials built from supergravity data are an important tool to investigating the stability of the equilibrium states under perturbations of the condensate.

Using the standard AdS/CFT dictionary it was proposed in [21] that the effective field theory potential induced by the scalar field is

$$
\mathcal{V}(\alpha)=\int_{0}^{\alpha}\left[\beta(\alpha)-\beta_{S}(\alpha)\right] d \alpha
$$

where $\beta(\alpha)$ are the boundary conditions and $\beta_{S}(\alpha)$ is the soliton line or a regularity condition that can be build as follow. Take a finite value of the scalar field at the origin and starting from this value shoot up towards infinity. The fall-off at infinity is fixed by the scalar field mass. The coefficients of the leading and subleading terms provide a point in the $(\alpha, \beta)$-plane. Repeat the operation several times and construct the line $\beta_{S}(\alpha)$. This prescription is such that the solitons are extremum of the effective field theory potential (5.1).

In the well-known 5-dimensional case when the boundary topology is $R \times S^{3}$, there is a non-vanishing Casimir energy and the vacuum gravitational energy of global $A d S_{5}$ can be exactly matched to the vacuum energy of the large $\mathrm{N}$ limit of $\mathcal{N}=4$ super Yang-Mills (see, 
e.g., [66]). The Hertog-Horowitz prescription also points out towards the identification of the gravitational energy and the energy of the field theory dual in designer gravity in the sense that the minimum energy solution is precisely the hairy soliton that corresponds to the minimum of the effective potential.

The prescription (5.1) is motivated by the fact that at finite temperature and $\alpha=0$, the gravitational solution is global AdS with zero mass. Therefore, a natural finite temperature generalization of (5.1) is as follows [57]. Take the Schwarzschild-AdS black at mass $M$. For large enough $M$, there are boundary conditions, $\beta_{M}(\alpha)$ that ensure the existence of a hairy black hole with the same mass. The finite temperature effective field theory potential is:

$$
\mathcal{V}_{M}(\alpha)=C \int_{0}^{\alpha}\left[\beta(\alpha)-\beta_{M}(\alpha)\right] d \alpha+M
$$

where $C$ is a positive constant that depends on the theory and allows to interpret $\mathcal{V}_{M}(\alpha)$ as the exact energy of the gravitational system. Note that there may be a critical value of the VEV where the hairy black hole ceases to exists and the line $\beta_{M}(\alpha)$ describe null or timelike singularities of the same mass. We exclude the singularities from our analysis. The hairy black holes of mass $M$ are indeed critical points of (5.2). The condition for hairy black hole stability is the convexity of the effective potential

$$
\beta^{\prime}\left(\alpha_{0}\right)-\beta_{M}^{\prime}\left(\alpha_{0}\right)>0
$$

The most stringent bound that we found in the previous section can be written as

$$
\beta^{\prime}\left(\alpha_{0}\right)+l^{2} \int_{z_{0}}^{0} U d z>0
$$

Hence, we conclude that

$$
-l^{2} \int_{z_{0}}^{0} U d z>\beta_{M}^{\prime}\left(\alpha_{0}\right)
$$

Note that we are able to obtain a bound on $\beta_{M}^{\prime}\left(\alpha_{0}\right)$ with our best estimate, given by the l.h.s. integral. The idea is that (5.3) represents the exact condition for stability. In the analysis of the previous section we have found an estimate that bounds its value. We do not claim that it is a sharp bound. Conversely, this opens the interesting scenario of providing sharp bounds for Schrödinger operators on the half-line determining the exact effective potential at finite temperature of a given field theory dual.

\section{Conclusions}

Let us explore in retrospective the linear stability of $A d S_{4}$. The relevant operator is $H=$ $-\partial_{z}^{2}$. $z$ belongs to the interval, $z \in\left[-\frac{\pi}{2} l, 0\right]$, where the boundary is at $z=0$. The regular perturbation with negative energy and generalized boundary conditions is $\psi(z)=$ $\sinh \left[\lambda\left(z+\frac{\pi l}{2}\right)\right]$. The relevant combination at the boundary is

$$
\frac{\psi^{\prime}(0)}{\psi(0)}=\lambda \frac{\cosh \left(\frac{\pi l}{2} \lambda\right)}{\sinh \left(\frac{\pi l}{2} \lambda\right)}>\frac{2}{\pi l} .
$$


In terms of the boundary condition of the scalar tensor theory we find $A d S_{4}$ instabilities for $-\beta^{\prime}(\alpha=0)>\frac{2 l}{\pi}$. Namely, linear vacuum stability is ensured whenever

$$
0 \leq \frac{2 l}{\pi}+\beta^{\prime}(\alpha=0)
$$

Going back to the discussion in the first paragraph of the introduction, and with the hindsight of our results, we see that it may very well be possible to have hairy black holes in theories where the vacuum is linearly stable. In particular, AdS invariant boundary conditions, $\beta=C \alpha^{2}$, satisfy trivially the bound (6.2). This makes the study of the general families of hairy black holes at the conformal mass indeed relevant.

We expect that the competition between the boundary condition and the effective potential of the master equation can be generalized to other cases, namely when the effective potential has a negative region. It may very well be possible as well that adequate boundary conditions could render the theory stable even when the effective potential is everywhere negative. This understanding is particularly relevant for gauged supergravity.

In four dimensions, there is the well known gauged $\mathcal{N}=8$ supergravity [67]. The mass of the 70 scalars, around the maximally supersymmetric $A d S_{4}$ vacuum, is $m^{2}=-2 l^{-2}$, see for instance table 2 in [68] and references therein, which reflects the fact that they are effectively massless around this vacuum. In a remarkable paper [71] it has been shown that the Nicolai-deWit gauged $\mathcal{N}=8$ supergravity is not unique, but a single member of a one parameter $(\omega)$ family of supergravities. As discussed in [72], two of the single scalar field truncations allow for a non-trivial $\omega$-deformation. For related work see also [73].

The four single-scalar field consistent truncations of the $\mathrm{SL}(8, \mathbb{R}) / \mathrm{SO}(8)$ sector, worked out in detail in [69], see also table 1 of [70], with a possible $\omega$-deformation, can be described by the scalar field self-interaction introduced in [30]. It also provide infinitely many examples that can be embedded in gauged $\mathcal{N}=1$ supergravity [39] and as recently remarked in [44] in gauged $\mathcal{N}=2$ supergravity with a Fayet-Iliopoulos term. We shall apply the methods discussed here to provide a throughout study of the hairy black hole stability in these theories in a future work.

\section{Acknowledgments}

A.A. thanks the enlightening discussions with Simon Ross about stability, Ioannis Papadimitriou for clarifying several aspects of the holographic interpretation and the hospitality of Durham University where this work was boosted. A.A. also thanks the the hospitality of Wellington Galleas at DESY and Jiří Bičák at Charles University where this work was continued and completed (supported from the Grant No. 14-37086G, Albert Einstein Center). We would like to thank Cristián Martinez and Raúl Rojas for interesting discussions and collaboration on related projects. Research of A. A. is supported in part by the Fondecyt Grants No 11121187 and 1141073, and by the Newton-Picarte Grants DPI20140053 and DPI20140115. Research of D.A. has been partially funded by the Fondecyt grants 1120446 and by the Newton-Picarte Grant DPI20140115. J.O. is supported by FONDECYT grant 1141073 and by the Newton-Picarte Grant DPI20140053. 
Open Access. This article is distributed under the terms of the Creative Commons Attribution License (CC-BY 4.0), which permits any use, distribution and reproduction in any medium, provided the original author(s) and source are credited.

\section{References}

[1] J.D. Bekenstein, Exact solutions of Einstein conformal scalar equations, Annals Phys. 82 (1974) 535 [INSPIRE].

[2] M. Heusler, A no hair theorem for selfgravitating nonlinear $\sigma$-models, J. Math. Phys. 33 (1992) 3497 [InSPIRE].

[3] D. Sudarsky, A simple proof of a no hair theorem in Einstein Higgs theory, Class. Quant. Grav. 12 (1995) 579 [inSPIRE].

[4] U. Nucamendi and M. Salgado, Scalar hairy black holes and solitons in asymptotically flat space-times, Phys. Rev. D 68 (2003) 044026 [gr-qc/0301062] [INSPIRE].

[5] A. Anabalon and J. Oliva, Exact hairy black holes and their modification to the universal law of gravitation, Phys. Rev. D 86 (2012) 107501 [arXiv:1205.6012] [INSPIRE].

[6] A. Anabalon, D. Astefanesei and R. Mann, Exact asymptotically flat charged hairy black holes with a dilaton potential, JHEP 10 (2013) 184 [arXiv:1308.1693] [INSPIRE].

[7] M. Cadoni and E. Franzin, Asymptotically flat black holes sourced by a massless scalar field, Phys. Rev. D 91 (2015) 104011 [arXiv: 1503.04734] [INSPIRE].

[8] K.A. Bronnikov and Yu. N. Kireev, Instability of black holes with scalar charge, Phys. Lett. A 67 (1978) 95 [inSPIRE].

[9] T.J.T. Harper, P.A. Thomas, E. Winstanley and P.M. Young, Instability of a four-dimensional de Sitter black hole with a conformally coupled scalar field, Phys. Rev. D 70 (2004) 064023 [gr-qc/0312104] [INSPIRE].

[10] G. Dotti, R.J. Gleiser and C. Martinez, Static black hole solutions with a self interacting conformally coupled scalar field, Phys. Rev. D 77 (2008) 104035 [arXiv:0710.1735] [INSPIRE].

[11] K.A. Bronnikov, J.C. Fabris and A. Zhidenko, On the stability of scalar-vacuum space-times, Eur. Phys. J. C 71 (2011) 1791 [arXiv:1109.6576] [InSPIRE].

[12] A. Anabalon and N. Deruelle, On the mechanical stability of asymptotically flat black holes with minimally coupled scalar hair, Phys. Rev. D 88 (2013) 064011 [arXiv:1307.2194] [INSPIRE].

[13] A. Anabalon, J. Bičák and J. Saavedra, Hairy black holes: stability under odd-parity perturbations and existence of slowly rotating solutions, Phys. Rev. D 90 (2014) 124055 [arXiv: 1405.7893] [INSPIRE].

[14] C.A.R. Herdeiro and E. Radu, Asymptotically flat black holes with scalar hair: a review, Int. J. Mod. Phys. D 24 (2015) 1542014 [arXiv: 1504.08209] [INSPIRE].

[15] C.A.R. Herdeiro and E. Radu, Kerr black holes with scalar hair, Phys. Rev. Lett. 112 (2014) 221101 [arXiv: 1403.2757] [INSPIRE].

[16] C. Herdeiro and E. Radu, Construction and physical properties of Kerr black holes with scalar hair, Class. Quant. Grav. 32 (2015) 144001 [arXiv:1501.04319] [INSPIRE]. 
[17] P. Breitenlohner and D.Z. Freedman, Positive energy in anti-de Sitter backgrounds and gauged extended supergravity, Phys. Lett. B 115 (1982) 197 [INSPIRE].

[18] P. Breitenlohner and D.Z. Freedman, Stability in gauged extended supergravity, Annals Phys. 144 (1982) 249 [INSPIRE].

[19] A. Ishibashi and R.M. Wald, Dynamics in nonglobally hyperbolic static space-times. 3. Anti-de Sitter space-time, Class. Quant. Grav. 21 (2004) 2981 [hep-th/0402184] [InSPIRE].

[20] G. Bonneau, J. Faraut and G. Valent, Selfadjoint extensions of operators and the teaching of quantum mechanics, Am. J. Phys. 69 (2001) 322 [quant-ph/0103153] [INSPIRE].

[21] T. Hertog and G.T. Horowitz, Designer gravity and field theory effective potentials, Phys. Rev. Lett. 94 (2005) 221301 [hep-th/0412169] [INSPIRE].

[22] T. Torii, K. Maeda and M. Narita, Scalar hair on the black hole in asymptotically anti-de Sitter space-time, Phys. Rev. D 64 (2001) 044007 [INSPIRE].

[23] M. Henneaux, C. Martinez, R. Troncoso and J. Zanelli, Black holes and asymptotics of $2+1$ gravity coupled to a scalar field, Phys. Rev. D 65 (2002) 104007 [hep-th/0201170] [INSPIRE].

[24] D. Sudarsky and J.A. Gonzalez, On black hole scalar hair in asymptotically anti-de Sitter space-times, Phys. Rev. D 67 (2003) 024038 [gr-qc/0207069] [INSPIRE].

[25] T. Hertog and K. Maeda, Black holes with scalar hair and asymptotics in $N=8$ supergravity, JHEP 07 (2004) 051 [hep-th/0404261] [INSPIRE].

[26] C. Martinez, R. Troncoso and J. Zanelli, Exact black hole solution with a minimally coupled scalar field, Phys. Rev. D 70 (2004) 084035 [hep-th/0406111] [INSPIRE].

[27] T. Kolyvaris, G. Koutsoumbas, E. Papantonopoulos and G. Siopsis, A new class of exact hairy black hole solutions, Gen. Rel. Grav. 43 (2011) 163 [arXiv:0911.1711] [INSPIRE].

[28] F. Correa, C. Martinez and R. Troncoso, Scalar solitons and the microscopic entropy of hairy black holes in three dimensions, JHEP 01 (2011) 034 [arXiv:1010.1259] [INSPIRE].

[29] A. Anabalon, F. Canfora, A. Giacomini and J. Oliva, Black holes with primary hair in gauged $N=8$ supergravity, JHEP 06 (2012) 010 [arXiv:1203.6627] [INSPIRE].

[30] A. Anabalon, Exact black holes and universality in the backreaction of non-linear $\sigma$-models with a potential in (A)dS $S_{4}$, JHEP 06 (2012) 127 [arXiv:1204.2720] [INSPIRE].

[31] A. Acena, A. Anabalon and D. Astefanesei, Exact hairy black brane solutions in AdS $S_{5}$ and holographic RG flows, Phys. Rev. D 87 (2013) 124033 [arXiv:1211.6126] [INSPIRE].

[32] A. Anabalon, Exact hairy black holes, Springer Proc. Phys. 157 (2014) 3 [arXiv:1211.2765] [INSPIRE].

[33] J. Aparicio, D. Grumiller, E. Lopez, I. Papadimitriou and S. Stricker, Bootstrapping gravity solutions, JHEP 05 (2013) 128 [arXiv: 1212.3609] [INSPIRE].

[34] L. Zhao, W. Xu and B. Zhu, Novel rotating hairy black hole in $(2+1)$-dimensions, Commun. Theor. Phys. 61 (2014) 475 [arXiv:1305.6001] [INSPIRE].

[35] H. Lü, Y. Pang and C.N. Pope, AdS dyonic black hole and its thermodynamics, JHEP 11 (2013) 033 [arXiv:1307.6243] [INSPIRE].

[36] P.A. González, E. Papantonopoulos, J. Saavedra and Y. Vásquez, Four-dimensional asymptotically AdS black holes with scalar hair, JHEP 12 (2013) 021 [arXiv:1309.2161] [INSPIRE]. 
[37] P. Bueno and C.S. Shahbazi, The violation of the no-hair conjecture in four-dimensional ungauged supergravity, Class. Quant. Grav. 31 (2014) 145005 [arXiv:1310.6379] [INSPIRE].

[38] A. Aceña, A. Anabalón, D. Astefanesei and R. Mann, Hairy planar black holes in higher dimensions, JHEP 01 (2014) 153 [arXiv:1311.6065] [inSPIRE].

[39] A. Anabalon and D. Astefanesei, Black holes in $\omega$-defomed gauged $N=8$ supergravity, Phys. Lett. B 732 (2014) 137 [arXiv:1311.7459] [inSPIRE].

[40] X.-H. Feng, H. Lü and Q. Wen, Scalar hairy black holes in general dimensions, Phys. Rev. D 89 (2014) 044014 [arXiv:1312.5374] [inSPIRE].

[41] X. Zhang and H. Lü, Exact black hole formation in asymptotically (A)dS and flat spacetimes, Phys. Lett. B 736 (2014) 455 [arXiv:1403.6874] [INSPIRE].

[42] P.A. González, E. Papantonopoulos, J. Saavedra and Y. Vásquez, Extremal hairy black holes, JHEP 11 (2014) 011 [arXiv:1408.7009] [INSPIRE].

[43] X. Zhang and H. Lü, Critical behavior in a massless scalar field collapse with self-interaction potential, Phys. Rev. D 91 (2015) 044046 [arXiv: 1410.8337] [INSPIRE].

[44] F. Faedo, D. Klemm and M. Nozawa, Hairy black holes in $N=2$ gauged supergravity, arXiv: 1505.02986 [INSPIRE].

[45] Z.-Y. Fan and H. Lü, Static and dynamic hairy planar black holes, Phys. Rev. D 92 (2015) 064008 [arXiv:1505.03557] [INSPIRE].

[46] Z.-Y. Fan and H. Lü, Charged black holes with scalar hair, JHEP 09 (2015) 060 [arXiv: 1507.04369] [INSPIRE].

[47] T. Hertog, Towards a novel no-hair theorem for black holes, Phys. Rev. D 74 (2006) 084008 [gr-qc/0608075] [INSPIRE].

[48] T. Faulkner, G.T. Horowitz and M.M. Roberts, New stability results for Einstein scalar gravity, Class. Quant. Grav. 27 (2010) 205007 [arXiv: 1006.2387] [INSPIRE].

[49] W. Boucher, Positive energy without supersymmetry, Nucl. Phys. B 242 (1984) 282 [INSPIRE].

[50] P.K. Townsend, Positive energy and the scalar potential in higher dimensional (super)gravity theories, Phys. Lett. B 148 (1984) 55 [InSPIRE].

[51] T. Regge and J.A. Wheeler, Stability of a Schwarzschild singularity, Phys. Rev. 108 (1957) 1063 [INSPIRE].

[52] F.J. Zerilli, Effective potential for even parity Regge-Wheeler gravitational perturbation equations, Phys. Rev. Lett. 24 (1970) 737 [InSPIRE].

[53] H. Kodama and A. Ishibashi, A master equation for gravitational perturbations of maximally symmetric black holes in higher dimensions, Prog. Theor. Phys. 110 (2003) 701 [hep-th/0305147] [INSPIRE].

[54] A. Ishibashi and H. Kodama, Stability of higher dimensional Schwarzschild black holes, Prog. Theor. Phys. 110 (2003) 901 [hep-th/0305185] [INSPIRE].

[55] E. Witten, Multitrace operators, boundary conditions and AdS/CFT correspondence, hep-th/0112258 [INSPIRE].

[56] I. Papadimitriou, Multi-trace deformations in AdS/CFT: exploring the vacuum structure of the deformed CFT, JHEP 05 (2007) 075 [hep-th/0703152] [INSPIRE]. 
[57] T. Hertog and G.T. Horowitz, Holographic description of AdS cosmologies, JHEP 04 (2005) 005 [hep-th/0503071] [INSPIRE].

[58] T. Hertog and S. Hollands, Stability in designer gravity, Class. Quant. Grav. 22 (2005) 5323 [hep-th/0508181] [INSPIRE].

[59] C. Martinez, Instability of three-dimensional conformally dressed black hole, Phys. Rev. D 58 (1998) 027501 [gr-qc/9801091] [INSPIRE].

[60] C. Martinez and J. Zanelli, Conformally dressed black hole in $(2+1)$-dimensions, Phys. Rev. D 54 (1996) 3830 [gr-qc/9604021] [INSPIRE].

[61] T. Hertog and K. Maeda, Stability and thermodynamics of AdS black holes with scalar hair, Phys. Rev. D 71 (2005) 024001 [hep-th/0409314] [INSPIRE].

[62] M. Henneaux, C. Martinez, R. Troncoso and J. Zanelli, Asymptotic behavior and Hamiltonian analysis of anti-de Sitter gravity coupled to scalar fields, Annals Phys. 322 (2007) 824 [hep-th/0603185] [INSPIRE].

[63] G. Dotti, Nonmodal linear stability of the Schwarzschild black hole, Phys. Rev. Lett. 112 (2014) 191101 [arXiv:1307.3340] [INSPIRE].

[64] A. Anabalon, D. Astefanesei and C. Martinez, Mass of asymptotically antide Sitter hairy spacetimes, Phys. Rev. D 91 (2015) 041501 [arXiv:1407.3296] [INSPIRE].

[65] B. Simon, The bound state of weakly coupled Schrödinger operators in one and two-dimensions, Annals Phys. 97 (1976) 279 [INSPIRE].

[66] V. Balasubramanian and P. Kraus, A stress tensor for Anti-de Sitter gravity, Commun. Math. Phys. 208 (1999) 413 [hep-th/9902121] [INSPIRE].

[67] B. de Wit and H. Nicolai, $N=8$ supergravity, Nucl. Phys. B 208 (1982) 323 [inSPIRE].

[68] G. Dall'Agata and G. Inverso, On the vacua of $N=8$ gauged supergravity in 4 dimensions, Nucl. Phys. B 859 (2012) 70 [arXiv:1112.3345] [InSPIRE].

[69] M. Cvetič, S.S. Gubser, H. Lü and C.N. Pope, Symmetric potentials of gauged supergravities in diverse dimensions and Coulomb branch of gauge theories, Phys. Rev. D 62 (2000) 086003 [hep-th/9909121] [INSPIRE].

[70] I. Papadimitriou, Non-supersymmetric membrane flows from fake supergravity and multi-trace deformations, JHEP 02 (2007) 008 [hep-th/0606038] [INSPIRE].

[71] G. Dall'Agata, G. Inverso and M. Trigiante, Evidence for a family of $\mathrm{SO}(8)$ gauged supergravity theories, Phys. Rev. Lett. 109 (2012) 201301 [arXiv:1209.0760] [INSPIRE].

[72] J. Tarrío and O. Varela, Electric/magnetic duality and RG flows in $A d S_{4} / C F T_{3}$, JHEP 01 (2014) 071 [arXiv: 1311.2933] [INSPIRE].

[73] Y. Pang, C.N. Pope and J. Rong, Holographic RG flow in a new $\mathrm{SO}(3) \times \mathrm{SO}(3)$ sector of $\omega$-deformed $\mathrm{SO}(8)$ gauged $N=8$ supergravity, JHEP 08 (2015) 122 [arXiv:1506.04270] [INSPIRE]. 